\title{
O PAPEL DO MÉDICO NA VISÃO DA SOCIEDADE DO SÉCULO XXI: O QUE REALMENTE IMPORTA AO PACIENTE?
}

\section{THE ROLE OF THE PHYSICIAN IN THE VIEW OF 21st CENTURY SOCIETY: WHAT DOES REALLY MATTERS TO THE PATIENT?}

\section{ANDREY BIFF SARRIS ${ }^{1}$; CARLOS RORY PUCCI FILHO'; CAROLINE DOBIS GRIK'; LETÍCIA CAROLLYNE GALVÃO'; RODRIGO DIEGO DE SOUZA²}

\author{
1 - Acadêmicos de Medicina da Universidade Estadual de Ponta Grossa (UEPG) (Ponta \\ Grossa, PR, Brasil). \\ 2 - Professor Assistente da Universidade Estadual de Ponta Grossa (UEPG) (Ponta Grossa, \\ PR, Brasil). \\ Autor para correspondência: andreybiff@hotmail.com
}

\section{RESUMO}

A formação histórico-social levou a figura do médico como detentor de conhecimento e da opinião final. O papel do médico para com a sociedade só logrará ser agente transformador quando consciente e pró ativo. Com o crescimento da validação e da legitimidade social adquiridos, permitiu-se, ao profissional, a livre autonomia e livre conduta. A formação exclusivamente técnica e a hierarquia da relação médico-paciente ficaram para trás para uma boa prática médica, o uso racional de procedimentos e medicamentos. A qualidade do serviço é avaliada conforme a percepção do cliente, neste caso, o paciente. Cada paciente possui uma perspectiva diferente e todas as avaliações são influenciadas e dependem do conhecimento, da necessidade, dos interesses e dos valores de cada um. O ideal seria que o próprio paciente tivesse voz dentro de determinados limites pois estes são a única fonte e o único objetivo dos serviços médicos prestados. Importa aos médicos serem conscientes dos papéis dinâmicos e importantes que a sociedade atual.

Palavras-chave: Relações Médico-Paciente; História da medicina; Assistência Médica; Ética Médica.

\section{ABSTRACT}

The historical-social formation made the figure of the doctor as holder of knowledge and decision maker. The role of the doctor towards society can only become a transforming agent when conscious and proactive. With the growth of validation and social legitimacy acquired, the medical professional was allowed free autonomy and free conduct. Nevertheless, the exclusively technical training and the hierarchy of the doctor-patient relationship are behind us for good medical practice the rational use of procedures and medications. The quality of the service is evaluated according to the client's perception, in this case, the patient. Each patient has a different perspective and all evaluations are influenced and depend on the knowledge, needs, interests and values of each patient. Ideally, the patient himself should have a voice, within certain limits, since these are the only source and sole purpose of medical services rendered. It is important for physicians to be aware of the dynamic and important roles of society today.

Keywords: Physician-Patient Relations; History of Medicine; Medical Assistance; Ethics, Medical. 


\section{INTRODUÇÃO}

Até alguns anos atrás, a sociedade estabelecia diversas atribuições aos médicos, muitas vezes, transformando-os em verdadeiros deuses. A capacidade de interação e intervenção do médico no ambiente social, seja em condutas em saúde ou nas demais, tinha um poder assombroso. Entretanto, especialmente nos últimos anos, o embate entre o papel místico do médico e a realidade fizeram dissolver laços e alterar a formação e a percepção dos médicos levando a graus diferentes de estresse físico-emocional. (MACHADO, 1997; RAMOS-CERQUEIRA \& LIMA, 2002). O desenvolvimento e aprimoramento da ética aplicada à medicina tornou as relações mais estáveis e niveladas. Isso, de um certo modo, permitiu que os interesses do paciente ganhassem luz frente aos interesses médicos. Entre os principais princípios éticos discorridos nos últimos tempos, citam-se: beneficência, não-maleficência, sigilo, autonomia e justiça. Sendo assim, esperase que o médico faça e pretenda o bem (beneficência), sem acarretar o mal, mesmo sem intenção (não-maleficência), de forma a respeitar o sigilo e autonomia do paciente - tudo isso em um contexto buscando justiça. (BAEROE, 2017). Com os problemas enfrentados pelo sistema público de saúde brasileiro e a dinâmica (por vezes, perversa) da maquinaria privada de assistência à saúde. (URDAN, 2001), fica a possível pergunta: diante do papel do médico na visão da sociedade, o que realmente importa ao paciente?

\section{METODOLOGIA}

Foi realizada consulta à literatura médica através das pesquisas nas bases de dados eletrônicos "MEDLINE", "LILACS" e "SCIELO" durante os meses de fevereiro e março de 2017. Nas bases, foram utilizadas os descritores "medicina" e "sociedade", "médico" e "sociedade", "médico" e "paciente", "visão do médico" e "visão do paciente" com os descritores nas línguas portuguesa e inglesa. Foram lidos os primeiros cem títulos de cada base, sendo lidos os resumos dos títulos considerados adequados à temática, ou seja, que enfatizavam as relações de médico e paciente, especialmente com aspectos voltados para a ética médica e as opiniões dos pacientes em relação ao profissional médico. Foram selecionados os que mantiveram esta adequação, sendo revisados quanto à manutenção da temática. 


\section{DISCUSSÃO}

\subsection{A formação do ideal médico}

A preocupação com a saúde física sempre foi motivo de inquietação da humanidade. O conhecimento de a natureza lhe ser fonte e principal fornecedora de insumos para tal fim acompanha esse princípio. O surgimento das medicinas oriental e ocidental foram distintos, sendo que em muitos momentos ocorreu uma grande permuta recíproca médica. (ALMEIDA FILHO, 2006).

O papel do médico acompanhou o desenvolvimento da civilização ao longo dos séculos. $\mathrm{Na}$ Grécia, o papel do médico era amplo, podendo o mesmo atuar no âmbito social, político, cultural e ético. Os herdeiros de Hipócrates rapidamente modificaram parte de tais preceitos por uma medicina mercadológica de cura na prática individual. Na Roma antiga os médicos eram escravos gregos valiosos a serviço da corte, do exército e de famílias nobres. Na idade média, ocorreu a anulação dessa pluripotencialidade do médico, da qual o algoz limitante foi a herança individualista e curativa da prática médica. (ALMEIDA FILHO, 2006).

O nascimento da ciência moderna libertou a figura do médico de entraves políticos, econômicos e sociais. Assim, a prática médica e o papel do médico na sociedade ganhou diversidade e novas potencialidades, apesar de preponderarem os focos individual e curativo à saúde humana em detrimento do coletivo. O Relatório Flexner, em 1910, deixou marcas de difícil superação, apesar de ser um desafio à geração médica do século XXI ressignificar esses valores, multiplicando as potencialidades de uma medicina cientifica e social na interação salutar entre individual e coletivo, privado e público, biológico e social, curativo e preventivo. (ALMEIDA FILHO, 2006).

O ideário de "grande profissão" dado à medicina está ligado a diversos fatores. Podem ser citados, com grande importância, a capacidade quase que única da carreira médica em ter realizado, ao longo da história, uma notável relação e aliança com o Estado (concedendo-Ihe prerrogativas legais para seu exercício exclusivo) e com a elite (vendendoIhe serviços particulares a preço de mercado). Isso permitiu a construção de uma profissão sólida, com garantias e proteções externas e com um caráter, até então, de retenção do conhecimento. (MACHADO, 1997). Ter liberdade de pensar, de agir e de estipular o valor monetário de seus serviços é o preceito fundamental de uma típica atividade liberal. E a estrutura de Estado e elite supracitada permitiu a consolidação desta prática liberal, que é 
a profissão médica. Todo esse processo de formação histórico-social levou a figura do médico como detentor de conhecimento e da opinião final. Para que isso ocorresse, foi necessário um alto grau de legitimidade dos dois lados (médico e paciente). De acordo com WEBER (1982), o sistema de dominação será tanto mais eficaz quanto maior a sua legitimidade. É preciso, afirma Weber, que as pessoas aceitem e reconheçam a autoridade, o poder e, consequentemente, admitam a dominação.

O processo de autonomia da atividade médica fora imprescindível para decisões e condutas que melhor se adaptassem a cada cliente. O papel do médico para com a sociedade só logrará ser agente transformador quando consciente e pró ativo. Esse processo, que parece estar se diluindo no século XXI, é fruto da imposição social que a profissão assumiu ainda no século XX. Com o crescimento da validação e da legitimidade social adquiridos, permitiu-se, ao profissional, a livre autonomia e livre conduta. (ARDIGÓ, 1999; MACHADO, 1997).

\subsection{A desmistificação e o desgaste da profissão médica}

A formação exclusivamente técnica e a hierarquia da relação médico-paciente ficaram para trás. A crescente disseminação do conhecimento e a importância do respeito à autonomia do paciente passaram a surgir como fatores principais na estruturação da prática de saúde. $\mathrm{O}$ acesso à informação transforma o paciente em um desafio para o médico. Perdeu-se a relação de subversão e o figural paterno, necessitando de novos meios para criação de uma relação médico-paciente eficaz. (BRASIL, 2009).

O papel do médico no século XXI está relacionado a uma atuação intersetorial. Considerando o panorama da medicina atual, o profissional médico necessita ser consciente de suas potencialidades, detentor de conhecimento tecnológico e biológico, ciente da importância de figurar nas várias instâncias gestoras e decisórias na macro e micropolítica social, bem como assumir papéis de cuidador, comunicador e educador na prática cotidiana. (BRASIL, 2009).

A eficiência dos resultados passa a depender não apenas da técnica e da experiência, mas de um entendimento pleno entre o doente e o médico, valorizando não somente o restabelecimento da saúde física e mental, mas o encaixe social de ambos dentro de suas funções. (ARDIGÓ, 1999; MACHADO, 1997).

Outro fator contribuinte para esse processo é a alteração do centro do cuidado em estratégicas de promoção e prevenção de saúde, não mais apenas curativas. Isso 
determina a presença do médico (especialmente o médico de família) no contexto social local, na possibilidade de transformação dos processos de adoecimento e de assistência à saúde. (ARDIGÓ, 1999; MACHADO, 1997).

Somados aos fatores técnicos, um outro problema perceptível é que a quebra ou a relação médico-paciente mal estruturada implicará em resultados terapêuticos diferentes. Entre os fatores que contribuíram (e contribuem) para o processo de desgaste da profissão médica, podem ser citados a criminalização do ato médico, a "judicialização da medicina", o sensacionalismo dos meios de comunicação, a livre opinião nas redes sociais, os precários sistemas de saúde, os defeitos na formação acadêmica e a má prática de alguns profissionais. (FRANZEN, 2008; MELLO, 2008). A criminalização do ato médico, ou seja, a possibilidade de o paciente, ao se sentir insatisfeito com determinada conduta ou complicação em seu tratamento, ao constatar qualquer irregularidade, denuncia 0 profissional em decorrência daquilo que identifica como erro médico, muitas vezes, é errônea, de má-fé e leva a um processo de receio na conduta por parte dos médicos. A isso, associa-se o processo de "judicialização", no qual, esses possíveis erros ou insatisfações se tornam processos jurídicos com cunho não apenas moral, mas financeiro. O sensacionalismo, por outro lado, pode ser visto nos pré-julgamentos, muitas vezes, realizados pela mídia (especializada ou não), levando à difamação não apenas de determinado indivíduo, mas com ampliação para toda a classe. O mesmo hoje se reflete em redes sociais, nas quais cada paciente sente-se no direito de expressar sua opinião, da forma como for. Por fim, a dificuldade na incorporação da abordagem desses fatores durante a graduação médica e má prática de alguns profissionais, acabam por dar voz ao processo de desgaste do médico. (COSTA, 2005; FRANZEN, 2008; MELLO, 2008).

O médico da atualidade deve ser consciente do papel dinâmico que apresenta na sociedade atual. Devem entender que são agentes positivos de mudança em qualquer situação, imbuídos dos elementos potenciais que lhe são prerrogativas, transformando dor em alívio, sofrimento em consolo, doença em reparação, limitação em reabilitação, iniquidade em justiça, injustiça em acerto, ausência em presença fraterna, dúvida em verdade. O médico transforma a sociedade quando faz de seus papéis atos de mudança positiva, individual ou coletivamente. (BRASIL, 2009).

A demanda complexa de práxis cotidiana da medicina, de pressões políticas, mercadológicas, e classistas, de condições de trabalho e de remuneração, a inadequada formação dos médicos nos cursos de graduação e a inabilidade, ou a falta de recursos pessoais e subjetivos, para exercer a contento esses papéis podem gerar situação de 
adoecimento do próprio profissional a práticas aberrantes e criminosas. (GOUVEIA, 2004).

\subsection{A "judicialização da medicina"}

O termo judicialização da medicina vem se tornando usual, pois cada vez mais indivíduos recorrem à Justiça para obter aportes necessários para seus tratamentos. Ou seja, a expressão aponta para problemas no acesso a bens e serviços da área da saúde, cirurgias não previstas pelo SUS, responsabilidade médica, liberação de leitos de unidades de terapia intensiva, medicamentos, dentre muitos outros, por intermédio de ações judiciais. (D'ESPINDULA, 2013).

Como as ações judiciais implicam conteúdos técnicos e médicos diversos e abrangentes, cria-se um conflito entre várias ideologias (a médica, a da gestão da saúde e a do Juízo), no qual a balança pende para o poder médico. Assim, a determinação judicial do fornecimento dos medicamentos pode ou não ter benefícios para a qualidade de vida do requerente ou para a população geral assistida, devido a falta de tempo para o judiciário avaliar previamente a real necessidade. Além disso, é renegado pelo judiciário a solicitação de outras opiniões técnicas quanto à dispensação dos medicamentos. (D'ESPINDULA, 2013; GADELHA, 2014).

Pelo poder médico, estes profissionais devem estar consultando sempre publicações especializadas e frequentando eventos científicos. Porém deve estar atento às ações da mídia em favor da indústria farmacêutica, que quase sempre interessada em comercializar inovações terapêuticas, culminando por exercer alguma influência sobre seus padrões de prescrição. Estudos concluem que a maioria das prescrições que chegam à Justiça é oriunda de hospitais universitários, que costumam avaliar novas tecnologias e tratamentos. (PEPE, 2010).

As vantagens da judicialização da medicina implicam em induzir a atualização de programas e protocolos de práticas terapêuticas. Como resultados negativos, em vários casos, uma precoce incorporação de medicamentos, visando reduzir as demandas judiciais, por vezes sem atender adequadamente aos critérios de eficácia, segurança e prioridades sanitárias. (D'ESPINDULA, 2013).

Para uma boa prática médica e o uso racional de procedimentos e medicamentos, contam a sua seleção adequada, a melhor conduta para o caso em pauta e o porte tecnológico da instituição em que se dá o atendimento, bem como a observância às diretrizes e protocolos estabelecidos com base em rigorosa busca da literatura científica 
especializada e resultante de criteriosa análise técnico-científica, que considera todas as alternativas disponíveis, em termos de resultado, segurança e custo-efetividade. (GADELHA, 2014).

\subsection{A qualidade de atendimento de acordo com o paciente - o que realmente importa!}

A relação médico-paciente também se trata de uma prestação de serviços, e assim como as demais áreas de atuação, a qualidade do serviço é avaliada conforme a percepção do cliente, neste caso, o paciente. Os profissionais médicos brasileiros estão inseridos em uma realidade em que precisam enfrentar diversos desafios, a começar pelas limitações e dificuldades no sistema público de saúde, a concorrência entre os demais profissionais, o nível de exigência dos pacientes que tem sido crescente, além das questões pessoais e interpessoais relacionadas a capitalização da medicina. (URDAN, 2001).

Ao avaliar a qualidade de um serviço, as pesquisas buscam salientar a Qualidade Percebida pelo cliente. Esta se trata de uma avaliação subjetiva na qual é confrontada a expectativa gerada previamente com relação ao serviço e o que de fato é ofertado, portanto, as interpretações pessoais e os processos psicológicos de transferências levam a diferentes percepções e experiências por parte dos clientes, sendo que cada um exprime sua visão individual da realidade (GARVIN, 1984). O modelo genérico da qualidade de serviços proposto com GRONROOS em 1990 coloca a Qualidade Percebida pelo cliente como um resultado comparativo entre a expectativa e a experiência vivenciada, sendo considerada boa quando os serviços superam ou ao menos alcançam as expectativas. Além disso, dois tipos de qualidades são considerados: qualidade técnica e qualidade funcional. A qualidade técnica seria o que foi prestado de serviço, enquanto que a qualidade funcional, de maneira mais subjetiva, seria como esse serviço foi prestado. Os serviços de saúde possuem suas particularidades, mas alguns dos princípios genéricos da qualidade de serviços nas demais áreas podem ser transferidos para a área da saúde e são úteis no auxílio do entendimento das avaliações feitas pelos pacientes. (BERWICK et al, 1990; DUKE, 2000; GRONROOS, 1990; JUN, 1998).

Porém, algumas singularidades se destacam, como a falta de clareza na conexão direta entre entrada e saída, devido à necessidade de tempo que o atendimento à saúde tem para que os resultados dos serviços prestados sejam avaliados, além disso, existe a dificuldade dos pacientes em avaliar os aspectos técnicos dos serviços, a divisão de autoridade que ocorre entre os médicos com os demais profissionais envolvidos no 
atendimento em âmbito hospitalar e a diversidade de clientes inseridos na esfera do profissional médico - o próprio paciente, a família envolvida, a comunidade de um modo geral, outros profissionais da área e também o conselho da sua classe. (BERWICK, 1990; DUKE, 2000; JUN, 1998).

Em se tratando de saúde, a qualidade percebida ainda abrange a necessidade do paciente de que o médico ofereça uma medida abrangente que venha garantir o bem-estar e o melhor desfecho possível dentro do processo saúde-doença. E essa qualidade também compreende o domínio técnico e o domínio interpessoal, sendo semelhante ao modelo proposto por Gronroos, de modo que o técnico compreende a aplicação da Ciência Médica e da sua tecnologia ao atender o paciente e conduzir o problema de saúde em questão, enquanto que o interpessoal refere-se a relação social e psicológica estabelecida entre médico e paciente, além de contar com outras amenidades como as características do ambiente onde o atendimento é prestado, levando em consideração pontos como conforto e cortesia, não apenas por parte do médico, como também dos recepcionistas e secretários do local. De um modo geral, os pacientes avaliam a qualidade técnica de forma indireta, pois na maioria das vezes não possuem um conhecimento amplo do domínio técnico, então a avaliação acaba sendo baseada na demonstração de interesse, na atenção desprendida e na preocupação do médico com o bem-estar e a saúde dos mesmos. Por outro lado, o domínio interpessoal é facilmente avaliado, levando-se em consideração as condições e amenidades sob as quais o cuidado é prestado e o tratamento que recebe por parte do médico (DONABEDIAN, 1980; GRONROOS,1990). Importante ressaltar que a avaliação de um dos domínios, quer seja positiva ou negativa, acaba por interferir no outro de modo a influenciar nos resultados, de modo que um domínio é dependente do outro, sendo que apenas o bom tratamento não é suficiente para a cura de uma doença orgânica, mas a aplicação da técnica por si só também pode não ser o suficiente, por isso diz-se que o próprio médico exerce um efeito placebo na condição do paciente que está sendo atendido. (DONABEDIAN, 1980; GRONROOS,1990).

Por muito tempo o domínio da técnica foi pilar único na formação do estudante de medicina, no entanto, a qualidade interpessoal tornou-se um desafio, fazendo com que as escolas de medicina de uma forma geral tenham mudado seus currículos, acrescentando disciplinas e instruindo professores a realçarem a necessidade de reumanização do médico, a importância da comunicação e da necessidade de olhar o paciente como um todo ao invés de um órgão ou um problema de forma isolada. (CLARK, 2001).

A qualidade nos serviços de saúde, neste caso específico, do atendimento médico, 
é mais facilmente avaliada nos consultórios, pois é onde ocorre grande parte do trabalho da classe médica e onde normalmente são estabelecidas relações mais duradouras. Um estudo realizado por BROWN E SWARTZ (1989) nos grupos da Universidade Federal de Minas Gerais, considerou 43 atributos como importantes no resultado da qualidade percebida pelos pacientes, a partir desta consideração, um questionário de satisfação foi aplicado a 1.050 pacientes. Os resultados deste estudo mostraram que seis fatores se destacam nas avaliações feitas pelos pacientes - as interações do médico e diagnóstico, a competência profissional, as interações de auxiliares, a comodidade de horários e de localização, o profissionalismo e responsabilidade profissional e comunicações auxiliares. Dentro desses, enquadram-se as 43 variáveis que irão esmiuçar esses fatores, e estas revelam pontos como: a necessidade dos pacientes de serem ouvidos, de terem informações sobre a sua saúde, o tempo dedicado pelo médico ao atendimento, a confiança inspirada pelo médico, a demonstração de interesse, atenção e respeito, a explicação dada pelo médico para ter optado por determinada conduta. A avaliação também leva em consideração pontos da questão financeira, sendo que os pacientes revelam não gostar quando percebem que o médico prioriza o dinheiro e esperam que os médicos sejam honestos ao cobrar por uma consulta e que também levem em consideração a situação financeira dos seus pacientes, não indicando medicamentos, exames ou demais procedimentos sem sua real necessidade. Outros pontos como o tempo de espera para conseguir um horário, a disponibilidade, o tempo de espera no momento da consulta e o atendimento recebido pelos recepcionistas durante esse intervalo, também possuem influencia na percepção do atendimento pelos pacientes. Este estudo, bem como os demais realizados dentro dessa área, evidencia que é um grande desafio o atendimento nos sistemas de saúde. (BROWN E SWARTZ, 1989; GRONROOS, 1990).

É indiscutível que os pacientes são a razão de existir qualquer sistema de prestação de serviços de saúde, mas eles consideram uma infinidade de fatores no momento do atendimento, comprometendo o índice de sucesso em atingir o objetivo final de proporcionar uma atenção integral ao seu paciente. Cada paciente possui uma perspectiva diferente e todas as avaliações são influenciadas e dependem do conhecimento, da necessidade, dos interesses e dos valores de cada um. Isso mostra que a chance de o médico errar ao tentar prever o que o paciente espera é normalmente muito grande, então o ideal seria que o próprio paciente tivesse voz, dentro de determinados limites (principalmente no que diz respeito a técnica), pois estes são a única fonte e o único objetivo dos serviços médicos prestados. (BROWN E SWARTZ, 1989; GRONROOS, 1990; 
PARASURAMAN, 1985).

\section{CONCLUSÃO}

Partindo do princípio de que o campo médico ultrapassa os limites da ciência, abrangendo os fatores experimentais, culturais, sociais e econômicos, e justapondo a essas a complexidade da relação interpessoal, da família, da mente associada ao corpo, já não cabe uma visão da medicina apenas no âmbito da racionalidade.

Importa aos médicos serem conscientes dos papéis dinâmicos e importantes que a sociedade atual, do século XXI, tanto carece a fim de serem agentes positivos de mudanças em qualquer situação, imbuídos dos elementos potenciais que the são prerrogativa, transformando dor em alívio, sofrimento em consolo, doença em reparação, limitação em reabilitação, iniquidades em justiça, perdas em possibilidades, injustiças em acertos, ausência em presença fraterna, dúvida em verdade, e tantas outras possibilidades.

\section{REFERÊNCIAS}

ARDIGÓ, A. Corso di Sociologia Sanitaria I ScuoladiSpecializzazione inSociologia Sanitaria. Bologna: Universitàdi Bologna, 1995. ApudCAPRARA, Andrea; FRANCO, Anamélia Lins e Silva. A RelaçãoPaciente-Médico: para uma Humanização da Prática Médica [online].Rio de Janeiro: Fundação Oswaldo Cruz, Escola Nacional de SaúdePública, Cadernos de Saúde Pública, v.15, n.3. Set. de 1999. In: ScieloPublic Health. Disponível em <http://www.scielosp.org/> [consulta; 10/02/2017].

ALMEIDA FILHO, N.; ROUQUAYROL, M. Z. História da Epidemiologia. In: Introdução à Epidemiologia. 4.ed. Rio de Janeiro: Guanabara Koogan, 2006. p.08-31

ARDIGÓ, A. Corso di Sociologia Sanitaria I Scuola di Specializzazione in Sociologia Sanitaria. Bologna: Universitàdi Bologna, 1995. ApudCAPRARA, Andrea; FRANCO, Anamélia Lins e Silva. A Relação Paciente-Médico: para uma Humanização da Prática Médica [online].Rio de Janeiro: Fundação Oswaldo Cruz, Escola Nacional de Saúde Pública, Cadernos de Saúde Pública, v.15, n.3. Set. de 1999. In: Scielo Public Health. Disponível em <http://www.scielosp.org/> [consulta; 10/02/2017]. 
BAEROE, Kristine, IVES, Jonathan, DE VRIES, Martine, SCHILDMAN, Jan. On classifying the field of medical ethics. BMC Medical Ethics, v.18, n.30, 2017.

BERWICK, Donald M., GODFREY, A. Blanton, ROESSNER, Jane. Curing health care - new strategies for quality improvement: a report on the National Demonstration Project on Quality Improvement in Health Care. San Francisco: Jossey-Bass, 1990.

BRASIL. Ministério da educação. Conselho Nacional de Educação. Diretrizes Curriculares Nacionais do Curso de Graduação em Medicina. 2009.

BROWN, Stephen W., SWARTZ, Teresa A. A gap analysis of professional service quality. Journal of Marketing, v.53, n.2, p.92-98, apr 1989.

CLARK, Liana R. How do we get back our humanity? Medical Economics, v. 78, n. 9, p. 9698, May 2001.

COSTA, Marcos Gutemberg Fialho da. Medicina Defensiva - Uma Necessidade. Publicação Oficial do Conselho Federal de Medicina, Brasília, Ano XX, n. 157, Nov./ dez. de 2005. Fala Médico, p. 16.

D'ESPINDULA, Thereza Cristina de Arruda Salomé. Judicialização da medicina no acesso a medicamentos: reflexões bioéticas. Revista. Bioética, Brasília, v.21, n. 3, p. 438-447, Dec. 2013.

DONABEDIAN, Avedis. Explorations in quality assessment and monitoring. Ann Arbor: Health Administration Press, 1980. V. I: The definition of quality and approaches to its assessment.

FRANZEN, Cláudio Balduíno. Em Nome do Interesse Público. Publicação Oficial do Conselho Federal de Medicina, Brasília, Ano XXIII, n. 174, Nov./dez. de 2008. Ética em Debate, p.10.

GADELHA, Maria Inês Pordeus. O papel dos médicos na judicialização da saúde. Revista CEJ, v. 18, n. 62, p. 65-70, jan/abr 2014. 
GARVIN, David A. What does "product quality" really means. Sloan Management Review, v. 26 , n. 1, p. 23-43, Fall 1984.

GOUVEIA, Valdiney Veloso. CONSELHO FEDERAL DE MEDICINA. O médico e o seu trabalho. Brasília, DF: Conselho Federal de Medicina, 2004.

GRONROOS, C. Service Quality: The Six Criteria of Good Service Quality. Review of Business. New York: St. John's University Press, 1990.

MACHADO, M. H. Os médicos no Brasil: um retrato da realidade. [online]. Rio de Janeiro:FIOCRUZ, 1997. 244 p.

MELLO, Luiz Eduardo Barbalho de. Intranquilidade e Dano à Credibilidade.Publicação Oficial do Conselho Federal de Medicina, Brasília, AnoXXIII, n. 174, Nov./ dez. de 2008. Ética em Debate, p.10.

PARASURAMAN, A., ZEITHAML, Valarie A., BERRY, Leonard L. A conceptual model of service quality and its implications for future research. Journal of Marketing, v.49, n.4, p.4150, Fall 1985.

PEPE, Vera Lúcia Edais et al. Caracterização de demandas judiciais de fornecimento de medicamentos "essenciais" no Estado do Rio de Janeiro, Brasil. Caderno Saúde Pública, Rio de Janeiro, v. 26, n. 3, p. 461-471, Mar 2010.

RAMOS-CERQUEIRA, A. T. A., LIMA, M. C. The establishment of the physician's identity: implications forundergraduate medical teaching, Interface - Comunic, Saúde, Educ, v.6, n.11, p.107-16, 2002.

URDAN, A. T. A qualidade de serviços médicos na perspectiva do cliente. Revista de Administração de Empresas, v.41, n.4, p.44-55, out/dez 2001.

WEBER, M. Ensaios de sociologia. Trad: Waltensir Dutra. 5.ed. Rio de Janeiro:Guanabara,1982. 